\title{
Antitumor effects of brucine immuno-nanoparticles on hepatocellular carcinoma in vivo
}

\author{
JIANMIN QIN ${ }^{1,2}$, LIN YANG $^{1}$, XIA SHENG ${ }^{1}$, ZHONGQIU SA $^{1,2}$, TAO HUANG $^{1}$, QI LI $^{2}$, \\ KEPAN GAO $^{3}$, QINGHUA CHEN ${ }^{3}$, JINGWEI MA ${ }^{4}$ and HEBAI SHEN ${ }^{4}$ \\ ${ }^{1}$ Department of General Surgery, The Third Hospital, The Second Military Medical University, Shanghai 201805; \\ ${ }^{2}$ Department of General Surgery, Putuo Hospital, Shanghai University of Traditional Chinese Medicine, Shanghai 200062; \\ ${ }^{3}$ National Pharmaceutical Engineering Research Center, Shanghai Institute of Pharmaceutical Industry, Shanghai 200040; \\ ${ }^{4}$ Department of Physical Chemistry, Shanghai Normal University, Shanghai 200234, P.R. China
}

Received June 12, 2016; Accepted October 12, 2017

DOI: $10.3892 / \mathrm{ol} .2018 .8168$

\begin{abstract}
In vitro and in vivo studies have demonstrated that brucine is able to inhibit the proliferation of liver cancer cells and growth of animal tumors, and may be a promising anticancer drug. However, high toxicity, poor water solubility, short half-life, narrow therapeutic window, and similar therapeutic and toxic doses limit its clinical application in the treatment of malignant tumors. In our previous study, brucine immuno-nanoparticles were successfully prepared and added to the culture medium of liver cancer SMMC-7721 cells, and the results indicated that the brucine immuno-nanoparticles were able to target the cell membrane of liver cancer SMMC-7721 cells and significantly inhibit the proliferation, adhesion, invasion and metastasis of SMMC-7721 cells. The aim of the present study was to investigate the antitumor effect of brucine immuno-nanoparticles in vivo by establishing an in situ transplanted liver cancer in nude mice. The results indicated that in vivo application of the brucine immuno-nanoparticles resulted in temporary liver and kidney damage, and significantly reduced the $\alpha$-fetoprotein (AFP) secretion of tumor cells (Bru-NP-MAb vs. the other groups; $\mathrm{P}<0.05)$. The brucine concentration of tumor tissues in the brucine immuno-nanoparticles group was significantly increased compared with that of the brucine nanoparticles group (Bru-NP-MAb vs. Bru-NP group or brucine group; $\mathrm{P}<0.05)$. The brucine immuno-nanoparticles were able to inhibit tumor growth and cluster of differentiation 34 expression and angiogenesis of tumor tissues, and induce the apoptosis of tumor cells (Bru-NP-MAb vs. Bru-NP group
\end{abstract}

Correspondence to: Professor Jianmin Qin, Department of General Surgery, The Third Hospital, The Second Military Medical University, 700 North Moyu Road, Jiangding, Shanghai 201805, P.R. China

E-mail: jianminqin@yahoo.com

Key words: hepatocellular carcinoma, brucine, nanoparticle, drug targeting, treatment or brucine group; $\mathrm{P}<0.05)$. In conclusion, as a novel type of targeted drug, brucine nanoparticles combined with anti-AFP monoclonal antibodies was more effective compared with brucine nanoparticles or brucine alone in inhibiting tumor growth via the enhancement of apoptosis, and the suppression of proliferation and angiogenesis in vivo. Therefore, the brucine immuno-nanoparticle is a promising targeted drug for the treatment of hepatocellular carcinoma.

\section{Introduction}

Incidence of hepatocellular carcinoma (HCC) ranks fifth among all malignant tumors. HCC is the second leading cause of cancer-associated mortality in the world. The overall 5 -year survival rate of liver cancer is $<5 \%$ (1). HCC is often accompanied by various degrees of cirrhosis, which accounted for between 70-90\% of all cases in China in 2003 (2). As a result of cirrhosis, the patients with HCC have poor tolerance to drug therapy and have a high risk of surgical resection (3). Although tumor recurrence may be prevented by systemic and local chemotherapy to increase the postoperative survival rate, to a certain extent, following hepatectomy or liver transplantation, current chemotherapeutics have poor sensitivity and poor targeting and therapeutic effects (3). The severe systemic toxic effects and side effects result in functional damage of important organs, including the heart, liver and kidney (4). The causes limit the extensive application of current chemotherapeutics. HCC is drug resistant to the majority of chemotherapeutics, and the therapeutic effect is $\sim 15 \%$ following systemic administration (2). Drug resistance may be overcome by increasing the effective drug concentration of tumor tissue, and the therapeutic effect can be increased $(3,4)$. Brucine is a weak indole alkaloid with poor water solubility. It is one of the antitumor drugs that has been studied in recent years. The proliferation of human hepatoma SMMC-7721 cells was inhibited following the administration of brucine in vitro, which indicated that the inhibition rate increased as the drug concentration of brucine increased (5). At a brucine concentration of $320 \mu \mathrm{g} / \mathrm{ml}$, the inhibition rate of cell proliferation was close to $100 \%$ (5). Deng et al (6-8) revealed that brucine was able to induce programmed cell death, caspase- 9 proteolysis 
and mitochondrial membrane depolarization of HepG2 cells to kill liver cancer cells. Brucine was able to inhibit the tumor growth of mice with solid tumors, to a certain extent, and stimulate and facilitate the hematopoietic system and immune system, and restore the damage of liver and kidney function caused by Heps tumor inoculation (7). The results demonstrated that brucine was beneficial to the hematopoietic and immune systems of mice with solid tumors, and may be a novel and promising antitumor drug.

Brucine is limited in its clinical application for malignant tumors owing to its high toxicity, poor water solubility, narrow therapeutic window, and similar toxic and therapeutic doses.

Nanoparticles (NPs) may be engineered to carry insoluble or highly toxic drugs using nanotechnology. When nano-drugs are applied in vivo, the antitumor drugs may be carried to the tumor tissues selectively, which results in an increased regional drug concentration and slow release of drugs, and maintains drug effects for a long time, whereas the drug concentration is lower than that of the non-tumorous tissues and other organs $(9,10)$. The research and development of nano-drugs expand the clinical application of highly toxic anticancer drugs (11).

In our previous study (12), brucine immuno-nanoparticles were successfully created by nanotechnology, and the results revealed that the brucine immuno-nanoparticles were stable, and exhibited even size distribution and a slow-releasing effect. The brucine immuno-nanoparticles were able to specifically combine with liver cancer cells, target the liver cancer cell membrane and exert its antitumor effects following in vitro application for the liver cancer SMMC-7721 cells. Brucine immuno-nanoparticles were able to inhibit the proliferation of liver cancer SMMC-7721 cells in a time- and dose-dependent manner. Compared with brucine and brucine nanoparticles, the brucine immuno-nanoparticles exhibited a more specific targeting for tumor cells, increased local drug concentration and effectively inhibited cancer cell proliferation, matrix adhesion, invasion and metastasis (12). Therefore, the present study investigated the distribution and antitumor effects of brucine immuno-nanoparticles in vivo by establishing an in situ liver cancer model in nude mice.

\section{Materials and methods}

Materials. Brucine (batch no., 110706-200 505; purity, >99\%; Chengdu Must Bio-Technology Co., Ltd., Chengdu, China), 5-fluorouracil (5-FU; Shanghai Xudong Haipu Pharmaceutical Co., Ltd., China; batch no., 090315), carboxylated poly(ethylene glycol) (PEG)-poly(lactic acid) (PLA) block copolymer (PLA-PEG-COOH; cat. no., PA20100302; molecular mass, 40 kDa; Jiangsu PegBio Co., Ltd., Jiangsu, China), mouse anti-human $\alpha$-fetoprotein (AFP) monoclonal antibody (MAb) (molecular mass, 70 kDa; Hangzhou HuaAn Biotechnology Co., Ltd., Hangzhou, China), brucine nanoparticles and brucine immuno-nanoparticles (The brucine immuno-nanoparticles were prepared by the National Pharmaceutical Engineering Research Center, Shanghai Institute of Pharmaceutical Industry and Department of Physical Chemistry, Shanghai Normal University), mass spectrometer (3200 Q Trap tandem mass spectrometer; Applied Biosystems; Thermo Fisher Scientific, Inc., Waltham, MA, USA), the liquid chromatography system
(SIL-HTC, LC-20AD and DGU-20A3; Shimadzu Corporation, Kyoto, Japan), automatic biochemical analyzer (Bayer AG, Leverkusen, Germany), abdominal 9.0 MHZ B-type ultrasonography (Prosound F75; Hitachi, Ltd., Tokyo, Japan), mouse anti-human Ki-67 MAb (cat. no., P6834; Sigma-Aldrich; Merck KgaA, Darmstadt, Germany), mouse anti-human CD34 Mab (cat. no., ab187282; Abcam, Cambridge, UK), citrate antigen retrieval buffer and diaminobenzidine (DAB) chromogenic kit (Fuzhou Maixin Biotech Co., Ltd., Fuzhou, China) for immunohistochemistry, terminal deoxynucleotidyl transferase dUTP nick-end labeling apoptosis kits (Boehringer Mannheim GmbH, Mannheim, Germany), fluorescein isothiocyanate (FITC)-conjugated goat anti-mouse IgG (Shanghai Unitech Bio-Technology Co., Ltd.), human AFP ELISA kits (IBL International GmbH, Hamburg, Germany), human hepatoma SMMC-7721 cell line (Shanghai Institutes for Biological Sciences Cell Institute of the Chinese Academy of Sciences, Shanghai, China) and $300 \mathrm{BALB} / \mathrm{c} \mathrm{nu} / \mathrm{nu}$ male nude mice, weighing 16-20 g, from Shanghai B\&K Universal Group Limited [production license no. SCXK (Shanghai, China) 2008-0016] were from the suppliers specified. All nude mice were quarantined for 1 week before the start of the experiment. Mice were housed in an animal facility maintained on a $12 / 12 \mathrm{~h} \mathrm{light/dark}$ cycle, at a constant temperature of $23 \pm 1^{\circ} \mathrm{C}$ and relative humidity of $44 \pm 5 \%$, and were given free access to tap water and food.

Establishment of an in situ transplanted liver cancer model in nude mice. Human hepatoma SMMC-7721 cells in the exponential phase were collected and prepared for cell suspension. Cell suspension $\left(0.2 \mathrm{ml} ; 5 \times 10^{6}\right.$ cells $)$ was subcutaneously injected into the axilla of nude mice. Tumors formed following inoculation for 2 weeks. The tumor-bearing mice were sacrificed by exsanguination under deep isoflurane anesthesia $(1.5 \%)$ when the tumor diameter was $>1 \mathrm{~cm}$. Tumors were then dissected under sterile conditions and placed into RPMI-1640 culture medium (cat. no., C22400500BT; Gibco; Thermo Fisher Scientific, Inc.). The connective tissue was removed and the tumor tissue was cut into $2 \times 2 \times 2 \mathrm{~mm}$ pieces. $\mathrm{BALB} / \mathrm{c}$ nu/nu male nude mice were anesthetized with an intraperitoneal injection of $60 \mathrm{mg} / \mathrm{kg}$ pentobarbital sodium. Following abdomen disinfection, an abdominal midline incision $1 \mathrm{~cm}$ below the xiphoid was made, and the left lobe of the liver was exposed. A tunnel under the liver capsule was made with curved forceps, the tumor mass was seeded in the tunnel and the abdominal incision was then closed. When the tumor diameter was $>1 \mathrm{~cm}$ determined using ultrasound detection, the mice were randomly selected for use in the experiments. The experimental protocols were reviewed and approved by the Committee of Ethics on Animal Experiments of Shanghai University of Traditional Chinese Medicine (Shanghai, China); all the animal work procedures were approved by the Institutional Animal Care and Use Committee of ShanghaiUniversity of TraditionalChineseMedicine(Shanghai, China).

Grouping. According to the experimental requirements, five groups were set up: Normal saline group (NS), 5-FU group, brucine group, brucine nanoparticle group (Bru-NP) and brucine immuno-nanoparticle group (Bru-NP-MAb). 
Drug doses, blood and tissue specimen management. Normal saline, 5-FU, brucine, brucine nanoparticles or brucine immuno-nanoparticles were respectively used to treat the tumor-bearing mice of various groups via the tail vein. The dose of brucine in the brucine, Bru-NP and Bru-NP-MAb groups was $3.23 \mathrm{mg} / \mathrm{kg}$. The dose of $5-\mathrm{FU}$ was $20 \mathrm{mg} / \mathrm{kg}$ in the 5-FU group. Mice in the normal saline group received an equal volume of normal saline. Drugs were administered three times a week in each group. Following treatment of the tumor-bearing mice for 1, 3, 7, 14, 21 or 30 days, 10 mice from each group were sacrificed by exsanguination under deep isoflurane anesthesia (1.5\%). Tumor volume and weight were measured, $1 \mathrm{ml}$ peripheral blood was drawn through the inferior vena cava, and tissues from the heart, lung, spleen, stomach, intestine, brain, muscle, fat, kidney, liver cancer and para-carcinoma tissues were preserved at $-80^{\circ} \mathrm{C}$. The tumor tissues and para-carcinoma tissues were cut into $1 \times 1 \times 0.5 \mathrm{~cm}$ sections, and fixed in anhydrous methanol at $25^{\circ} \mathrm{C}$ for $24 \mathrm{~h}$, dehydrated in graduated concentrations of ethanol (70, 80, 90, $95,100 \%$ ) and embedded in paraffin for $4 \mu \mathrm{m}$ sections.

Measurement of the liver and kidney functions and AFP levels in peripheral blood. In each group, $100 \mu 1$ serum was taken. Using an automatic biochemical analyzer, liver and kidney function indices, including albumin, alanine aminotransferase (ALT), aspartate aminotransferase, alkaline phosphatase, blood urea nitrogen $(\mathrm{BUN})$ and creatinine $(\mathrm{Cr})$, were determined. In total, 10 samples at the indicated time were analyzed in each group. Anti-human AFP ELISA kits were used to determine the serum AFP level in each group.

Distribution of brucine immuno-nanoparticles in different tissues. On the third day of drug application, $0.2 \mathrm{~g}$ heart, lung, spleen, stomach, intestine, brain, muscle, fat, kidney and liver cancer tissue, and adjacent cancer tissue from tumor-bearing animals in each group were separately taken and homogenized. Homogenate $(100 \mu \mathrm{l})$ with $20 \mu \mathrm{l}$ marker $(1 \mu \mathrm{g} / \mathrm{ml})$ and $300 \mu \mathrm{l}$ methanol was agitated, and then centrifuged at $11,739 \times \mathrm{g}$ at $4^{\circ} \mathrm{C}$ for $3 \mathrm{~min}$. A $100 \mu \mathrm{l}$ volume of supernatant was used to determine the brucine content using liquid chromatography-mass spectrometry.

Determination of the degree of tumor necrosis. Paraffin sections of tumor tissue in each group at different time points were deparaffinized with xylene, rehydrated in a graded series of ethanol, stained with hematoxylin and eosin and mounted using neutral gum, and the pathological changes were then observed under a light microscope (magnification, $\mathrm{x} 40$ ). The mean necrotic area in each group was calculated according by 10 tissue slices

The degree of necrosis was evaluated as follows: Mild necrosis $(\leq 30 \%)$, moderate necrosis $(30-70 \%)$ and severe necrosis $(>70 \%)$.

Determination of CD34 and Ki-67 expression in tumor tissues. Paraffin sections of tumor tissue at different time points in each group were deparaffinized with xylene and rehydrated in a graded series of ethanol. The slices were then added to citrate antigen retrieval buffer and boiled in a microwave for $10 \mathrm{~min}$ to retrieve tissue antigens.
Sections were then rinsed three times with 0.01 M PBS for 5 min, and mixed with $50 \mu 1$ mouse anti-human CD34 Mab (cat. no. ab187282; dilution 1:1,000; Abcam) or $50 \mu 1$ mouse anti-human Ki-67 MAb (cat. no. P6834; dilution 1:1,000; Sigma-Aldrich; Merck KGaA). Sections were then incubated at $4^{\circ} \mathrm{C}$ in wet conditions for $12 \mathrm{~h}$ and at room temperature for $30 \mathrm{~min}$. The slices were then rinsed three times with $0.01 \mathrm{M}$ PBS for $5 \mathrm{~min}$. DAB solution was added for staining at room temperature for $3 \mathrm{~min}$, and $0.01 \mathrm{M}$ PBS was used to wash the slices. Hematoxylin was used to re-stain at room temperature for $10 \mathrm{sec}$, graduating dilutions of ethanol were used to dehydrate $(70,80,90,95$ and $100 \%)$, pure xylene was used for clearing and neutral gum was used to seal the slices. Finally, the slices were observed under a light microscope (magnification, x100).

Tumor cells with brown particles in nuclei were identified as $\mathrm{Ki}-67^{+}$cells and were observed under x100 magnification. The microvessels ( $<8$ red blood cell diameter) and single endothelial cell were enumerated under a light microscope (magnification, x100).

Determination of tumor cell apoptosis. Paraffin sections of tumor tissue at different time points in each group were deparaffinized in xylene, rehydrated in a graded series of ethanol and submerged in $3 \%$ hydrogen peroxide solution at room temperature for $10 \mathrm{~min}$, and were then washed three times with $0.01 \mathrm{M}$ PBS for $5 \mathrm{~min}$. Proteinase $\mathrm{K}$ solution $(50 \mu \mathrm{l} ; 20 \mu \mathrm{g} / \mathrm{ml})$ was added to the sample tissue at $37^{\circ} \mathrm{C}$ in a humidified chamber for $20 \mathrm{~min}$. Tissues were then washed three times with distilled water for $5 \mathrm{~min}$. Subsequently, $20 \mu 1$ labeling buffer was added to tissue samples at room temperature for $15 \mathrm{~min}$. Next, $20 \mu \mathrm{l}$ labeling buffer containing terminal deoxynucleotidyl transferase and biotin-11-dUTP (1:8) was added to the sample tissue at $37^{\circ} \mathrm{C}$ in a humidified chamber for $60 \mathrm{~min}$. The $20 \mathrm{X}$ saline sodium citrate (SSC) solution was diluted 10 times, the labeled tissue samples were immersed in 2X SSC at room temperature for $15 \mathrm{~min}$, and the samples were washed three times with $0.01 \mathrm{M}$ PBS for $3 \mathrm{~min}$. Confining liquid $(50 \mu \mathrm{l})$ was added to sample tissues at room temperature for $30 \mathrm{~min}$. The excess liquid was then removed, and $50 \mu \mathrm{l}$ confining liquid containing avidin-horseradish peroxidase (dilution, 1:50) was added to sample tissues, which were placed in a humidified chamber at $37^{\circ} \mathrm{C}$ for $60 \mathrm{~min}$. Subsequently, the samples were washed three times with $0.01 \mathrm{M}$ PBS for $3 \mathrm{~min}$. DAB was added for coloration for $3 \mathrm{~min}, 0.01 \mathrm{M}$ PBS was used to rinse, serial dilutions of ethanol were used to dehydrate $(70,80,90,95$ and 100\%), pure xylene was used for clearing and neutral gum was used to seal the slices. Finally, the samples were observed under a light microscope (magnification, x200).

Determination of tumor inhibition rate. The liver was completely removed and the tumor was completely dissected. The tumor size was determined using a Vernier caliper, and the longest and shortest tumor diameters were respectively recorded by millimeter unit.

Tumor size $V=a b^{2} / 2$ (a, length of tumor; $b$, width of tumor)

Tumor inhibition rate $(\%)=\left[1-\left(\mathrm{V}_{\text {treatment groups }} / \mathrm{V}_{\text {control group }}\right)\right] \times 100$ 
Table I. Serum $\alpha$-fetoprotein level of various groups at the indicated times.

\begin{tabular}{|c|c|c|c|c|c|c|}
\hline Group & 1 day & 3 days & 7 days & 14 days & 21 days & 30 days \\
\hline NS & $13.13 \pm 1.63$ & $13.08 \pm 1.69$ & $13.31 \pm 1.35$ & $13.41 \pm 1.96$ & $15.59 \pm 2.39$ & $16.32 \pm 3.21$ \\
\hline 5-FU & $14.06 \pm 2.05$ & $10.40 \pm 2.18^{\mathrm{a}, \mathrm{b}}$ & $11.48 \pm 2.56^{\mathrm{b}}$ & $10.74 \pm 1.38^{\mathrm{a}, \mathrm{b}}$ & $8.65 \pm 2.83^{\mathrm{a}, \mathrm{b}}$ & $10.02 \pm 4.23^{\mathrm{a}, \mathrm{b}}$ \\
\hline Brucine & $12.71 \pm 1.09$ & $8.96 \pm 2.71^{\mathrm{a}, \mathrm{b}}$ & $9.49 \pm 2.41^{\mathrm{a}, \mathrm{b}}$ & $13.44 \pm 1.96^{\mathrm{b}}$ & $13.78 \pm 1.96^{\mathrm{b}}$ & $15.33 \pm 2.11^{\mathrm{b}}$ \\
\hline Bru-NP & $13.67 \pm 1.36$ & $6.41 \pm 1.23^{\mathrm{a}}$ & $7.10 \pm 0.95^{\mathrm{a}}$ & $9.10 \pm 1.16^{\mathrm{a}, \mathrm{b}}$ & $11.23 \pm 2.53^{\mathrm{a}, \mathrm{b}}$ & $11.73 \pm 1.85^{\mathrm{a}, \mathrm{b}}$ \\
\hline Bru-NP-MAb & $12.26 \pm 1.33$ & $6.33 \pm 0.91^{\mathrm{a}}$ & $6.33 \pm 0.95^{\mathrm{a}}$ & $5.90 \pm 0.94^{\mathrm{a}}$ & $5.47 \pm 0.80^{\mathrm{b}}$ & $6.12 \pm 0.89^{a}$ \\
\hline
\end{tabular}

Results are presented as the mean \pm standard deviation concentrations in $\mu \mathrm{g} / \mathrm{l}(\mathrm{n}=10)$. ${ }^{\mathrm{a}}$ Compared with NS group $(\mathrm{P}<0.05)$. ${ }^{\mathrm{b}} \mathrm{Compared}$ with Bru-NP-MAb group $(\mathrm{P}<0.05)$. Bru-NP, brucine nanoparticle; MAb, monoclonal antibody; 5-FU, 5-fluorouracil; NS, normal saline.

Effect of brucine immuno-nanoparticles on the survival time in nude mice bearing tumors. A total of 20 tumor-bearing mice in each group were observed and the survival time following treatment was recorded. The dose of brucine in the brucine, Bru-NP and Bru-NP-MAb groups was $3.23 \mathrm{mg} / \mathrm{kg}$. The dose of $5-\mathrm{FU}$ was $20 \mathrm{mg} / \mathrm{kg}$ in the 5-FU group. Mice in the normal saline group received an equal volume of normal saline. Drugs were administered via the tail vein three times per week in each group. The diet, drinking and movement of all nude tumor-bearing mice in each group was observed twice a day during treatment. Survival time was defined as the interval between starting drug administration and final mortality as a direct result of the tumors.

Life-prolonging rate $(\%)=($ mean survival time in experimental groups/mean survival time in the control group-1) x 100

Statistical analysis. Statistical analysis was performed using SPSS statistics software (version 17.0; SPSS, Inc., Chicago, IL, USA). Data are expressed as the mean \pm standard deviation. A one-way analysis of variance was used to compare differences between groups at the same time point. The Levene test was used for variance homogeneity; the level of Levene test was $a=0.05$. The least significant difference test was adopted when variances were homoscedastic. The approximate F-test Welch method was employed and Dunnett's T3 method was used between groups when variances were not homoscedastic. $\mathrm{P}<0.05$ was considered to indicate a statistically significant difference.

\section{Results}

Identification of nude mice in an in situ transplanted liver cancer model. After 4 weeks of in situ tumor inoculation on the left lobe of the liver in nude mice, abdominal 9.0 MHZ B-type ultrasonography was used to detect the size and blood supply of the tumor. When the maximum tumor diameter was $>1 \mathrm{~cm}$, the tumor-bearing mice were randomly grouped for experiments.

Liver and kidney functions and AFP level in peripheral blood. On the 30th day of drug application, serum ALT level in the brucine group was increased compared with that of the Bru-NP-MAb group $(\mathrm{F}=3.996 ; \mathrm{P}<0.05 ; \mathrm{n}=10)$. On the 14th day, the serum BUN level was increased in the Bru-NP group when compared with the Bru-NP-MAb group $(\mathrm{F}=1.898 ; \mathrm{P}<0.05$; $\mathrm{n}=10$ ). On the 21st day, the serum $\mathrm{Cr}$ level was increased in the Bru-NP group when compared with the Bru-NP-MAb group $(\mathrm{F}=1.587 ; \mathrm{P}<0.05 ; \mathrm{n}=10)$.

Between the first day and the 14th day, AFP levels in the control group remained relatively stable and were increased slightly. On the seventh day, AFP levels in the Bru-NP-MAb group were significantly decreased compared with those of the brucine and 5-FU groups $(\mathrm{F}=17.027 ; \mathrm{P}<0.05 ; \mathrm{n}=10)$. On the 14th, 21st and 30th days, AFP levels were decreased significantly in the Bru-NP-MAb group when compared with the other treatment groups $(\mathrm{F}=16.977,16.425$ and 12.574, respectively; $\mathrm{P}<0.05 ; \mathrm{n}=10$; Table I).

Tissue distribution of brucine immuno-nanoparticles. After 3 days of drug application, no brucine was detected in the heart, lung, spleen, stomach, intestine, muscle, fat, kidney, tumors and adjacent tumor liver tissues in the brucine group. In the Bru-NP group, brucine was detected in the spleen, lung, kidney, muscle, tumor and adjacent tumor liver tissues. In the Bru-NP-MAb group, brucine was only detected in the spleen, tumor and adjacent tumor liver tissues. The brucine concentration of tumor tissues in the Bru-NP-MAb group was significantly increased compared with that of the Bru-NP group, and the brucine concentration of liver tissues near the tumor was significantly decreased compared with that of the Bru-NP group $(\mathrm{P}<0.05)$. The brucine concentration of lung, kidney and muscle tissues in the Bru-NP-MAb group was significantly lower than that of the Bru-NP group $(\mathrm{F}=520.792$ and 445.846, respectively; $\mathrm{P}<0.05 ; \mathrm{n}=10$ ). No significant difference in the brucine concentration of spleen tissues between the Bru-NP-MAb and the Bru-NP groups was identified ( $\mathrm{F}=0.004$; $\mathrm{P}>0.05 ; \mathrm{n}=10$ ) (Fig. 1).

Degree of tumor necrosis. After 14 days of drug application, the degree of tumor necrosis increased in the brucine, 5-FU and Bru-NP-MAb groups when compared with the normal saline group $(\mathrm{F}=6.299 ; \mathrm{P}<0.05 ; \mathrm{n}=10)$. After 30 days of drug application, the degree of tumor necrosis in the Bru-NP-MAb and 5-FU groups was more marked compared with that of the brucine and Bru-NP groups $(\mathrm{F}=17.769 ; \mathrm{P}<0.05 ; \mathrm{n}=10)$. On the 30 th day, the degree of tumor necrosis in the Bru-NP-MAb and 5-FU groups reached the peak, but there was no marked difference between the Bru-NP-MAb and 5-FU groups (Fig. 2). 


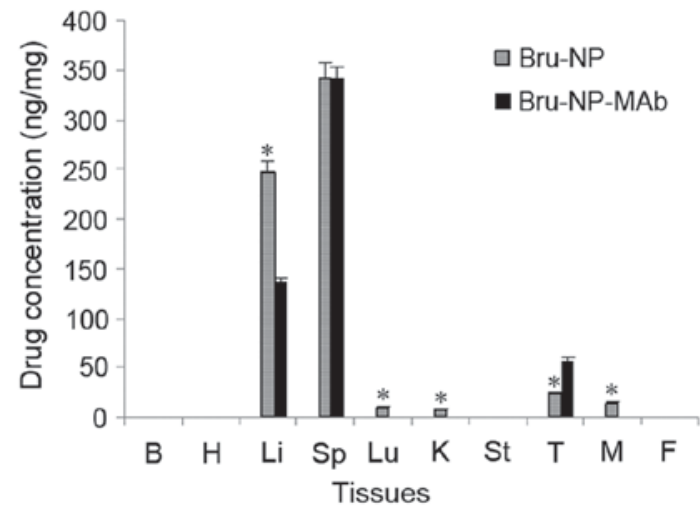

Figure 1. Tissue distribution of brucine following in vivo application for 3 days. ${ }^{*} \mathrm{P}<0.05$ vs. Bru-NP-MAb. B, brain; H, heart; Li, liver; Sp, spleen; Lu, lung; K, kidney; St, stomach; T, tumor; M, muscle; F, fat; Bru-NP, brucine nanoparticle; MAb, monoclonal antibody.

Expression of CD34 and Ki-67 in tumor tissues. The microvascular density (MVD) of tumor tissue was counted in 5 randomly selected visual fields using light microscopy (magnification, $\mathrm{x} 200$ ), and the vascular endothelial cells stained brown were considered positive for expression of CD34 or Ki-67 respectively. The percentage of MVD = the number of microvascular/visual field) $\mathrm{x} 100 \%$. The percentage of $\mathrm{Ki}-67$ expression $(\%)=(\mathrm{Ki}-67$ positive cell count $/$ total tumor cells) $\mathrm{x} 100 \%$. The mean of the MVD or Ki-67 expression in each group was calculated according to 10 tissue slices.

After 14 and 30 days of drug application, CD34 expression of the tumor tissues in the 5-FU group, the Bru-NP and Bru-NP-MAb group significantly decreased when compared with the normal saline group $(\mathrm{F}=3.230$ and 19.257; $\mathrm{P}<0.05 ; \mathrm{n}=10)$. The CD34 expression of the tumor tissue in the Bru-NP-MAb and 5-FU groups markedly decreased $(15.17 \pm 5.81$ cells/field vs. $16.50 \pm 6.38$ cells/field, Bru-NP-MAb group vs. 5-FU group). The CD34 expression of the tumor tissue among the 5-FU, Bru-NP and Bru-NP-MAb group was significantly different $(\mathrm{F}=9.257 ; \mathrm{P}<0.05 ; \mathrm{n}=10$; Fig. 3$)$.

After 1 day and 14 days, Ki-67 expression in tumor tissues decreased significantly in the 5-FU, Bru-NP and Bru-NP-MAb groups when compared with the normal saline group $(\mathrm{F}=4.546$; $\mathrm{F}=5.039 ; \mathrm{P}<0.05 ; \mathrm{n}=10)$. Ki-67 expression of tumor tissues in the Bru-NP-MAb group was decreased compared with that of the brucine group after 14 days $(\mathrm{F}=2.321 ; \mathrm{P}<0.05 ; \mathrm{n}=10)$. After 30 days, Ki-67 expression of tumor tissues in the brucine and Bru-NP groups was significantly increased compared with that of the Bru-NP-MAb group $(\mathrm{F}=18.937 ; \mathrm{P}<0.05 ; \mathrm{n}=10)$. As the time of drug application increased, 5-FU, brucine, brucine nanoparticles and brucine immuno-nanoparticles all noticeably inhibited the $\mathrm{Ki}-67$ expression in the tumor tissues. The inhibitory effect on the Ki-67 expression was most noticeable in the Bru-NP-MAb group (Fig. 4).

Tumor cell apoptosis. Cells were counted in 5 randomly selected visual fields using light microscopy (magnification, $\mathrm{x} 200$ ), and the cells stained brown were considered apoptotic cells. The apoptotic index of tumor cells = apoptotic cell count/total cell count $x 100 \%$. The mean of the apoptotic index in each group was calculated according to 10 tissue slices.

After 1, 14 and 30 days of drug application, the apoptotic indices of tumor cells in the brucine group, 5-FU, Bru-NP and Bru-NP-MAb groups were increased compared with the saline group $(\mathrm{F}=233.731, \mathrm{~F}=133.667$ and $\mathrm{F}=92.441$, respectively;
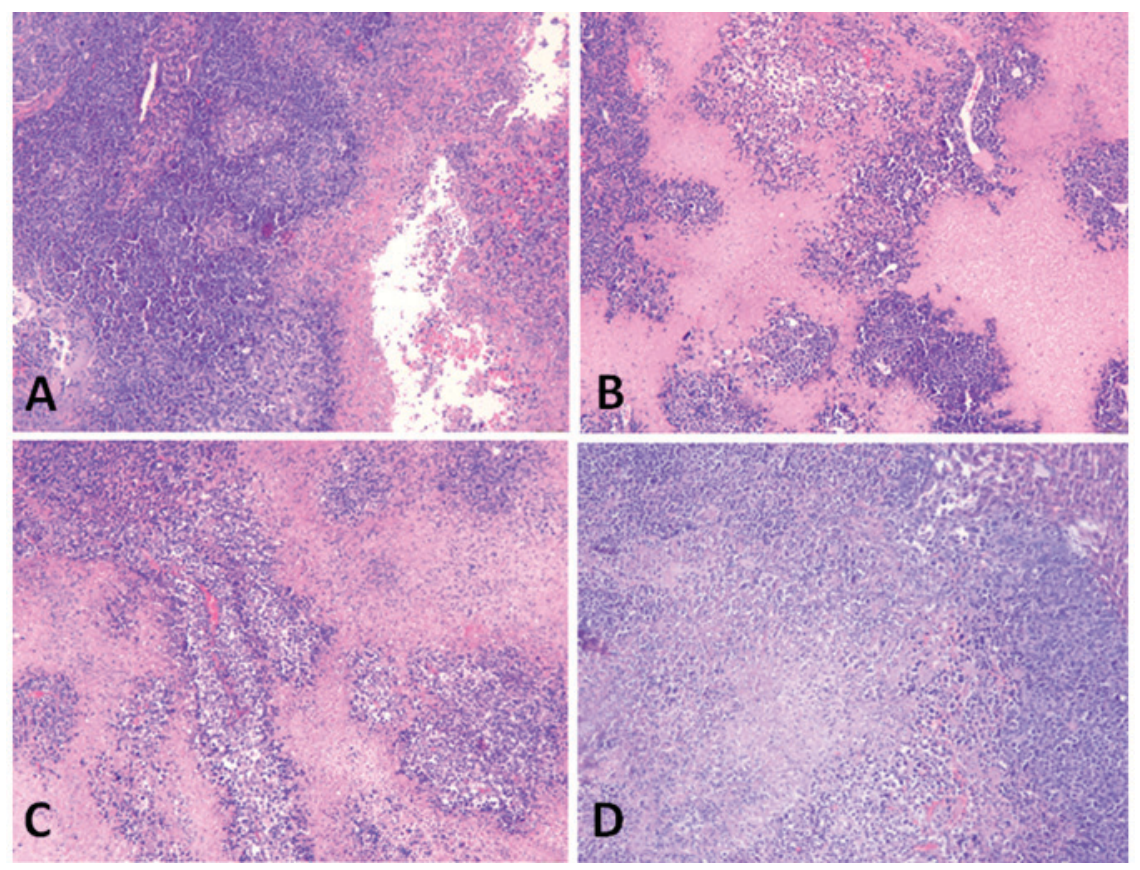

Figure 2. Tumor necrosis in various groups following in vivo application for 30 days (hematoxylin and eosin staining; magnification, $\mathrm{x} 40$ ). (A) Brucine group, $46.17 \pm 12.58 \%$ tumor necrosis. (B) Bru-NP group, $63.50 \pm 15.08 \%$ tumor necrosis. (C) Bru-NP-MAb group, $80.67 \pm 10.91 \%$ tumor necrosis. (D) 5-FU group, $70.50 \pm 10.33 \%$ tumor necrosis. The degree of tumor necrosis was more severe in treated groups compared with that of NS group; the degree of tumor necrosis in the Bru-NP-MAb and 5-FU groups was severe necrosis, but there was no significant difference between the Bru-NP-MAb and 5-FU groups ( $>0.05$ ). Bru-NP, brucine nanoparticle; MAb, monoclonal antibody; 5-FU, 5-fluorouracil; NS, normal saline. 


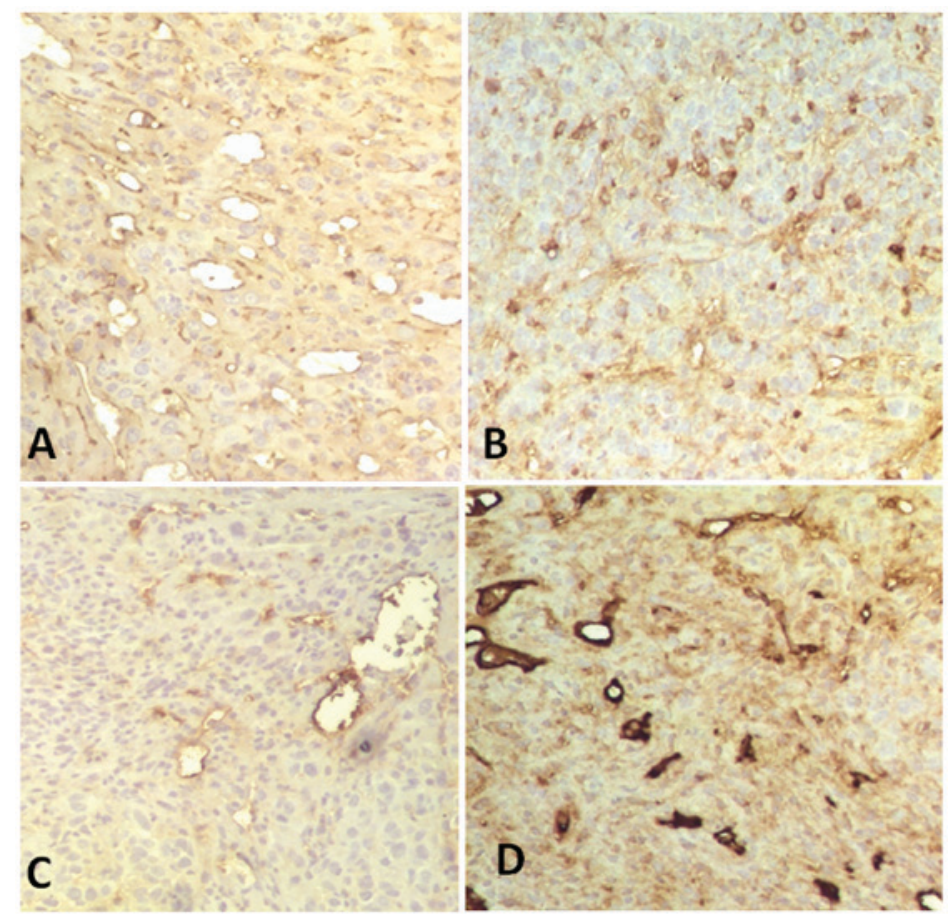

Figure 3. CD34 expression in tumor tissues in various groups following in vivo application for 30 days (immunohistochemical staining; magnification, x200). (A) Brucine group, $60.83 \pm 18.96 \%$ of $\mathrm{CD} 34^{+}$tumor cells of tumor tissues. (B) Bru-NP group, $32.17 \pm 12.12 \%$ of $\mathrm{CD} 34^{+}$tumor cells of tumor tissues. (C) Bru-NP-MAb group, $15.17 \pm 5.81 \%$ of CD34 $4^{+}$tumor cells of tumor tissues. (D) 5 -FU group, $16.50 \pm 6.38 \%$ of $\mathrm{CD}^{+} 4^{+}$tumor cells of tumor tissues. The number of $\mathrm{CD} 34^{+}$tumor cells in the Bru-NP-MAb and 5-FU groups was lower than that of the other groups, but there was no significant difference between the Bru-NP-MAb and 5-FU groups ( $\mathrm{P}>0.05$ ). Bru-NP, brucine nanoparticle; MAb, monoclonal antibody; 5-FU, 5-fluorouracil; CD34, cluster of differentiation 34.

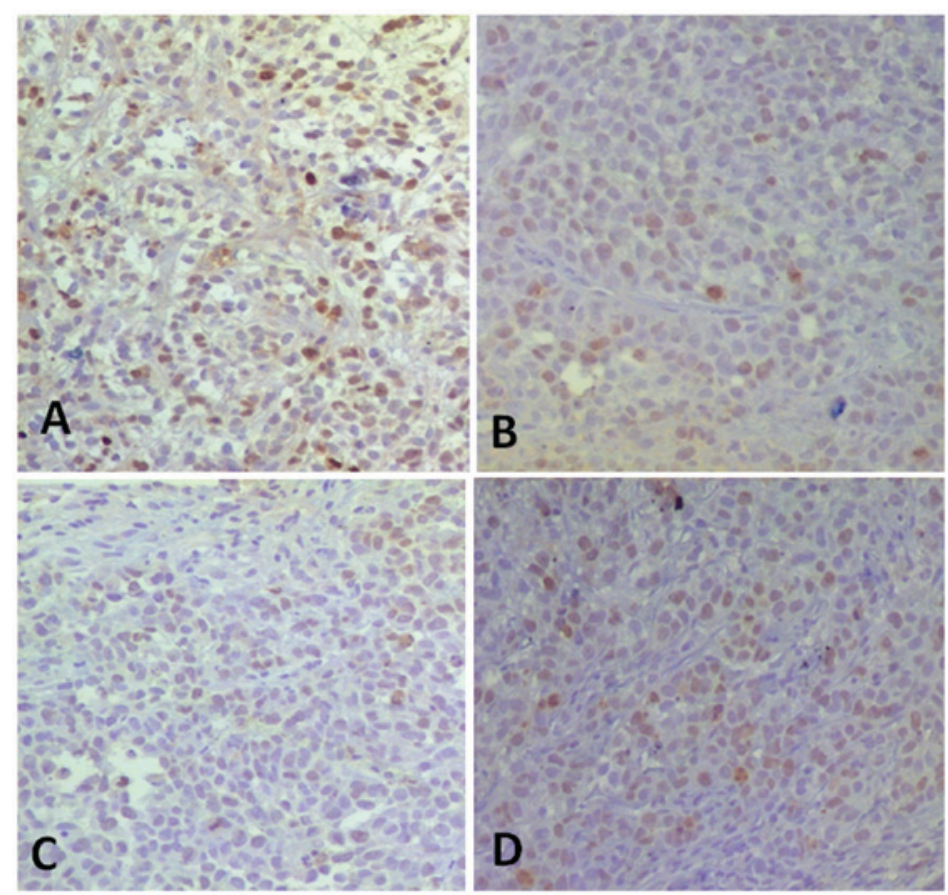

Figure 4. Ki-67 expression in tumor tissues in various groups following in vivo application for 30 days (immunohistochemical staining; magnification, x200). (A) Brucine group, $46.67 \pm 11.25 \%$ of CD34+ tumor cells of tumor tissues. (B) Bru-NP group, 35.50 $\pm .18 \%$ of CD34 tumor cells of tumor tissues. (C) Bru-NP-MAb group, 16.67 $\pm 8.16 \%$ of CD34 $4^{+}$tumor cells of tumor tissues. (D) 5 -FU group, $25.00 \pm 7.07 \%$ of CD34 tumor cells of tumor tissues. The number of $\mathrm{Ki}-67^{+}$tumor cells in the treated groups was less than that of the NS group, the number of Ki-67+ tumor cells in the Bru-NP-MAb and 5-FU groups was lower than that of the other groups, but there was no significant difference between the Bru-NP-MAb and 5-FU groups (P>0.05). Bru-NP, brucine nanoparticle; MAb, monoclonal antibody; 5-FU, 5-fluorouracil; CD34, cluster of differentiation 34; NS, normal saline.

all $\mathrm{P}<0.05 ; \mathrm{n}=10)$. The apoptotic index of tumor cells in the Bru-NP-MAb group was increased compared with that of the Bru-NP group $(\mathrm{P}<0.05 ; \mathrm{n}=10)$. On the first, 14th and 30th day, the apoptotic indices of tumor cells in the Bru-NP-MAb 


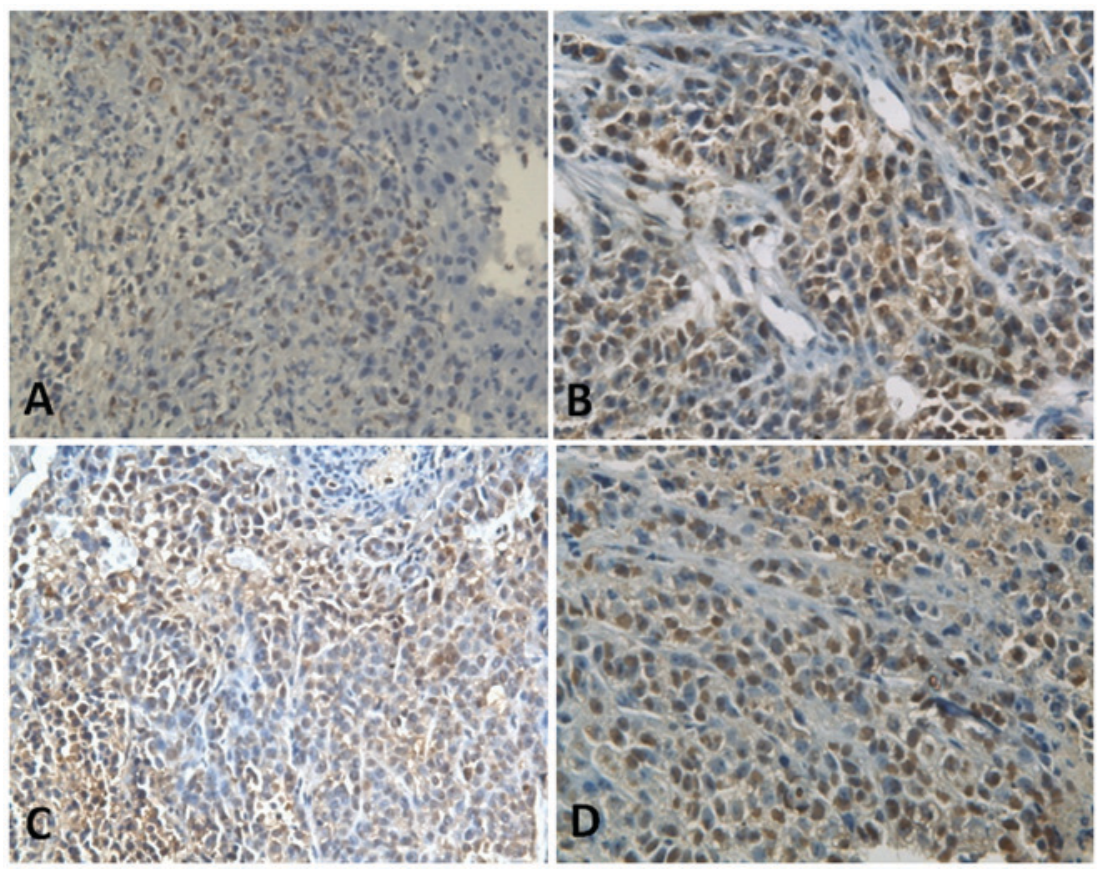

Figure 5. Apoptotic index of tumor cells in various groups after in vivo application for 1 day (immunohistochemical staining; magnification, $\mathrm{x} 200$ ). (A) Brucine group, $24.08 \pm 2.33 \%$ of apoptotic tumor cells of tumor tissues. (B) Bru-NP group, 33.59 $\pm 1.48 \%$ of apoptotic tumor cells of tumor tissues. (C) Bru-NP-MAb group, $53.22 \pm 3.23 \%$ of apoptotic tumor cells of tumor tissues. (D) 5-FU group, $27.87 \pm 3.13 \%$ of apoptotic tumor cells of tumor tissues. The apoptotic index of tumor cells in the treated groups was increased compared with that of the NS group, the apoptotic index of tumor cells was highest in the Bru-NP-MAb group, and there was a significant difference between the Bru-NP-MAb and other treatment groups $(\mathrm{P}<0.05)$. Bru-NP, brucine nanoparticle; MAb, monoclonal antibody; 5-FU, 5-fluorouracil; NS, normal saline.

Table II. Tumor volumes of various groups at the indicated times.

\begin{tabular}{|c|c|c|c|c|c|c|}
\hline Group & 1 day & 3 days & 7 days & 14 days & 21 days & 30 days \\
\hline NS & $224.17 \pm 18.15$ & $306.75 \pm 13.98$ & $564.08 \pm 42.94$ & $1,026.17 \pm 88.30$ & $1,688.50 \pm 79.08$ & $2,214.67 \pm 146.26$ \\
\hline $5-\mathrm{FU}$ & $219.33 \pm 15.10$ & $248.33 \pm 21.06^{\mathrm{a}, \mathrm{b}}$ & $414.17 \pm 20.73^{\mathrm{a}, \mathrm{b}}$ & $651.25 \pm 44.92^{\mathrm{a}, \mathrm{b}}$ & $758.5 \pm 53.91^{\mathrm{a}, \mathrm{b}}$ & $910.92 \pm 37.00^{\mathrm{a}, \mathrm{b}}$ \\
\hline Brucine & $227.17 \pm 13.96$ & $275.17 \pm 25.74^{\mathrm{a}}$ & $514.92 \pm 25.70^{\mathrm{a}, \mathrm{b}}$ & $833.75 \pm 73.89^{\mathrm{a}, \mathrm{b}}$ & $980.92 \pm 55.50^{\mathrm{a}, \mathrm{b}}$ & $1,171.92 \pm 59.57^{\mathrm{a}, \mathrm{b}}$ \\
\hline Bru-NP & $208.90 \pm 14.90$ & $298.00 \pm 17.54$ & $447.30 \pm 40.82^{\mathrm{a}, \mathrm{b}}$ & $768.00 \pm 61.06^{\mathrm{a}, \mathrm{b}}$ & $888.10 \pm 36.33^{\mathrm{a}, \mathrm{b}}$ & $1,074.40 \pm 64.21^{\mathrm{a}, \mathrm{b}}$ \\
\hline Bru-NP-MAb & $212.25 \pm 10.44$ & $275.75 \pm 10.47^{\mathrm{a}}$ & $356.92 \pm 21.20^{\mathrm{a}}$ & $464.17 \pm 21.45^{\mathrm{a}}$ & $515.33 \pm 32.50^{a}$ & $532.50 \pm 38.21^{\mathrm{a}}$ \\
\hline
\end{tabular}

Results are presented as the mean \pm standard deviation volumes in $\mathrm{mm}^{3}(\mathrm{n}=10)$. ${ }^{\mathrm{a} C o m p a r e d}$ with NS group $(\mathrm{P}<0.05)$. ${ }^{\mathrm{b}} \mathrm{Compared}$ with Bru-NP-MAb group $(\mathrm{P}<0.05)$. Bru-NP, brucine nanoparticle; MAb, monoclonal antibody; 5-FU, 5-fluorouracil; NS, normal saline.

group revealed the greatest increase, and were $53.22 \pm 3.23$, $40.97 \pm 3.18$ and $39.93 \pm 3.78 \%$, respectively (Fig. 5).

Tumor inhibition rate. After 3, 7, 14,21 and 30 days of drug application, the tumor size in the brucine group, 5-FU, Bru-NP and Bru-NP-MAb groups decreased significantly when compared with the normal saline group $(\mathrm{F}=7.715,35.252,59.825,389.271$ and 360.477, respectively; all $\mathrm{P}<0.05 ; \mathrm{n}=10$ ). After 7, 14, 21 and 30 days of drug application, tumor size decreased significantly in the Bru-NP-MAb group when compared with the brucine, 5-FU and Bru-NP groups ( $\mathrm{F}=35.252,59.825,389.271$ and 360.477 , respectively; all $\mathrm{P}<0.05$; $\mathrm{n}=10$; Table II). After 7, 14, 21 and 30 days of drug application, the tumor inhibition rates in the Bru-NP-MAb group were 36.73, 54.77, 69.48 and 75.96\%, respectively, and were significantly increased compared with that of the brucine, 5-FU and Bru-NP groups at the same time point $(\mathrm{F}=12.517,15.265,19.126$ and 26.524; $\mathrm{P}<0.05 ; \mathrm{n}=10)$. After 30 days of drug application, the tumor inhibition rate in the Bru-NP-MAb group was $75.96 \%$, and was significantly increased compared with that of the 5-FU, brucine and Bru-NP groups $(58.87,47.08$ and 51.49\%; $\mathrm{F}=26.524 ; \mathrm{P}<0.05 ; \mathrm{n}=10)$.

Survival time and life-prolonging rate of tumor-bearing animals. The survival time of tumor-bearing mice in the 5-FU, Bru-NP and Bru-NP-MAb groups was increased compared with that of the saline group $(\mathrm{F}=9.010 ; \mathrm{P}<0.05 ; \mathrm{n}=20)$. The survival time in the Bru-NP-MAb group was increased compared with that of the 5-FU, brucine and Bru-NP groups $(\mathrm{F}=11.210 ; \mathrm{P}<0.05 ; \mathrm{n}=20)$. Compared with the brucine, Bru-NP and 5-FU groups, the life-prolonging rate was the highest in the Bru-NP-MAb group (60.43 vs. $1.38,19.09$ and $25.2 \%$; $\mathrm{F}=12.160 ; \mathrm{P}<0.05 ; \mathrm{n}=20$; Table III). 
Table III. Survival times of animals with bearing tumor in various groups.

\begin{tabular}{lc}
\hline Group & Survival times, days \\
\hline NS & $50.80 \pm 12.30$ \\
$5-\mathrm{FU}$ & $63.60 \pm 16.26^{\mathrm{a}, \mathrm{b}}$ \\
Brucine & $50.10 \pm 11.19$ \\
Bru-NP & $60.50 \pm 12.54^{\mathrm{b}}$ \\
Bru-NP-MAb & $81.50 \pm 14.25^{\mathrm{a}}$ \\
\hline
\end{tabular}

Results are presented as the mean \pm standard deviation times $(n=20)$.

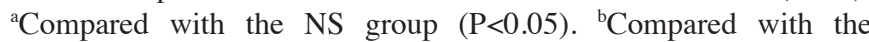
Bru-NP-MAb group $(\mathrm{P}<0.05)$. Bru-NP, brucine nanoparticle; MAb, monoclonal antibody; 5-FU, 5-fluorouracil; NS, normal saline.

\section{Discussion}

Chemotherapy is an important treatment for malignant tumors. A number of antitumor drugs of low molecular mass tend to spread evenly in the body, which results in relatively uniform tissue distribution (11). These drugs cannot distinguish tumor cells from normal cells, leading to a number of adverse effects, including bone marrow suppression and gastrointestinal toxicity, which limit their clinical application (11). In 1906, Enrilich first proposed the concept of targeted drug delivery, namely to selectively distribute drugs within the lesions so as to decrease the toxic side effect on normal tissues, to increase the drug concentration in the target tissue and to enhance the bioavailability of drugs. The most notable feature of targeted drugs is the ability to deliver the therapeutic drug to the target organ, increase the concentration of drugs in the target tissues, organs and cells, and prolong the time of drug effect (12). Thereby, targeted drugs are able to lead to a decrease in the dose, a decrease in the toxic side effect and an increase in drug efficiency (13). Targeted drug application is particularly suitable to certain antitumor drugs with an increased toxic side effect. Currently, the most common carriers are endogenous macromolecules or macromolecules through chemical synthesis, such as albumin, antibodies, glycoproteins, dextran or poly-amino acids, which are based on physical and chemical properties of carriers and target sites (14). Ideal carriers have high solubility, high stability, high purity, non-toxicity, biodegradability and low antigenicity (15).

PLA is a polymer with ideal biocompatibility and biodegradability, with metabolic end-products in vivo of carbon dioxide and water (15). PEG is a safe non-toxic hydrophilic polymer, often used as a protective agent of various types of microsphere (16). The PLA structure containing a large number of ester bonds has a decreased water solubility (16). Hydrophilic PEG chains and hydrophobic PLA chains may be used to prepare the PEG-PLA nanoparticles using physical and chemical techniques (16). The PEG-PLA nanoparticles have a marked hydrophilicity and are able to markedly enhance long-circulating and slow-release effects (16). PEG-PLA is an amphiphilic block copolymer with favorable biocompatibility and biosecurity (17). The block copolymers, as an effective drug carrier, have a long cycle, high bioavailability and low toxicity, and are able to automatically aggregate in aqueous solution to form a core-shell structure and bind the insoluble drug with the internal hydrophobic PLA chains, thereby increasing the drug solubility (17). The PEG-PLA block copolymers may be degraded into PEG and PLA, which are non-toxic and may be completely discharged (17). The PEG-PLA block copolymers are able to bind specific ligands to possess the active targeting of drug delivery and be evenly distributed in the body following in vivo application. Thus, the drugs carried by the PEG-PLA block copolymers may be delivered to specific targeted organs or tissues (18). As an effective carrier, the PEG-PLA block copolymers have clear advantages in targeted drug delivery, and have been considered as a preferred drug carrier in recent years (19).

As a drug carrier coupled with MAbs, which confer on the carrier high specificity and targeting, the drugs may be specifically delivered to tumor tissues where tumor cells produce a specific protein following carriage of antitumor drugs in vivo. The targeted drugs have a maximal antitumor effect and lower toxic side effect, which is regarded as one of the most promising drug therapies for malignant tumors in the future (20-22). Kan et al (23) prepared the human serum albumin (HAS) adriamycin (ADM) nanoparticles combined with HAb18 MAbs and obtained HAb18-HSA (ADM) nanoparticles. In vivo studies demonstrated that HAb18-HSA ADM nanoparticles exhibited an increased tumor inhibition rate compared with that of HSA ADM nanoparticles and ADM $(\mathrm{P}<0.001)$. Liu and Cai (24) used the dual-functional cross-linker succinimidyl 3-(2-pyridyldithio) propionate to couple the human liver cancer MAb HAb18 to prepare mitoxantrone-loaded albumin microspheres. In vitro study revealed that the immune microspheres were able to specifically accumulate around the human liver cancer SMMC-7721 cells and be internalized into the targeted cells, which results in slow drug release within cells and destruction of the SMMC-7721 cells.

In recent years, a number of studies $(25,26)$ indicated that brucine exerted marked inhibitory effects on tumor cell proliferation. In vitro studies demonstrated that brucine exerted, to a certain degree, an inhibitory effect on cell proliferation on stomach cancer SGC7901 and MGC803, lung cancer A549, colon cancer LoVo, liver cancer SMMC7721 and HepG2 cells. However, brucine is a highly toxic drug with a narrow therapeutic window, narrow safe range and short half-life. An excessive dose will easily cause central toxicity, including dizziness, headache and epileptic seizures, which limit its clinical application. To decrease the toxicity of brucine, Qu et al (27) used stearoyl ethanolamine-PEG 2000 to modify the surface of liposomes and developed brucine stealth liposomes. The results demonstrated that stealth liposomes were able to markedly enhance the antitumor effects of brucine. Wu et al (28) combined doxorubicin-poly(butyl cyanoacrylate) nanoparticles with the acid ferritin MAb and prepared liver-specific doxorubicin immuno-nanoparticles. Following accumulation of doxorubicin in the liver tumor, it is able to markedly increase the drug concentration, extend the time at which it is effective, enhance the efficacy and decrease the drug toxicity to other organs. As aforementioned, Kan et al (29) also demonstrated that the targeted drug exhibited more marked antitumor effects compared with single chemotherapy drug. 
AFP is a biomarker to detect hepatocellular carcinoma; the sensitivity and specificity of early diagnosis for $\mathrm{HCC}$ are 79 and $78 \%$, respectively (30-32). In order to decrease the toxicity of brucine, in our previous study (12), the carboxylated PEG-PLA copolymer was selected as a carrier material. Phacoemulsification technology was applied to prepare the brucine nanoparticles, and chemical coupling technology was applied to couple the brucine nanoparticles to anti-human AFP MAb to successfully prepare the brucine immuno-nanoparticles. In vitro studies indicated that the brucine immuno-nanoparticles had a uniform size distribution and were evenly distributed around the cell membrane of liver cancer SMMC-7721 cells. The results revealed that the brucine immuno-nanoparticles specifically accumulated in the cell membrane in a similar ring shape and inhibited the proliferation of human hepatoma SMMC-7721 cells (12).

In the present study, a nude mice liver cancer model was established by in situ inoculation technology. Following application of brucine immuno-nanoparticles in vivo for 3 days, brucine was not detected in every tissue in the brucine group. In the Bru-NP group, brucine was detected in the spleen, lung, kidney, muscle, tumor and liver tissues near the tumor. Brucine was only detected in the spleen, tumor and liver tissues near the tumor in the Bru-NP-MAb group. The drug concentration of brucine in the Bru-NP-MAb group was significantly increased compared with that of the Bru-NP group. The drug concentration of brucine in the liver tissues near tumor was significantly decreased compared that of the Bru-NP group. The results indicated that the brucine immuno-nanoparticles have promising slow-releasing and antitumor effects.

The results of the present study demonstrated that in vivo application of the brucine immuno-nanoparticles caused temporary liver and kidney function damage and significantly decreased the secretion of AFP by liver cancer SMMC-7721 cells. The brucine immuno-nanoparticles have good slow release and tumor targeting properties, and inhibit the expression of CD34 and angiogenesis of tumor tissues, induce tumor cell apoptosis and inhibit tumor growth. Furthermore, the brucine immuno-nanoparticles significantly prolonged the survival time of tumor-bearing mice.

In conclusion, the results of the present study demonstrated that in vivo application of brucine immune-nanoparticles is able to achieve delivery of brucine to tumor tissues and result in a continuous and slow release of the brucine within tumor tissues. The antitumor effect of the brucine immune nanoparticles was more marked compared with that of brucine, brucine nanoparticles and 5-FU. The brucine immune-nanoparticles are a promising targeted drug for HCC in the future. Further prospective study will involve preclinical trials to demonstrate the potential of clinical application of the targeted drug delivery system towards liver cancer therapy.

\section{Acknowledgements}

The present study was supported by the Shanghai Education Commission (grant no. 07CZ017), the Shanghai Science and Technology Commission (grant no. 1052nm060000) and the National Natural Science Foundation of China (grant no. 30873341).

\section{Competing interests}

The authors declare that they have no competing interests.

\section{References}

1. Parkin DM, Bray F, Ferlay J and Pisani P: Global cancer statistics, 2002. CA Cancer J Clin 55: 74-108, 2005.

2. Tang ZY, Ye SL, Liu YK, Qin LX, Sun HC, Ye QH, Wang L, Zhou J, Qiu SJ, Li Y, et al: A decade's studies on metastasis of hepatocellular carcinoma. J Cancer Res Clin Oncol 130: 187-196, 2004.

3. Liu CL and Fan ST: Nonresectional therapies for hepatocellular carcinoma. Am J Surg 173: 358-365, 1997.

4. Yang TS, Wang CH, Hsieh RK, Chen JS and Fung MC: Gemcitabine and doxorubicin for the treatment of patients with advanced hepatocellular carcinoma: A phase I-II tria1. Ann Oncol 13: 1771-1778, 2002.

5. Qin JM, Xu XJ, Sheng X, Li Q, Yin PH, Zhang M, Yang L and Sang ZQ: Anti-hepatocellular carcinoma study of the brucine in rats. Chin J Gen Surg 26: 219-221, 2011 (In Chinese).

6. Deng X, Yin F, Lu X, Cai B and Yin W: The apoptotic effect of brucine from the seed of Strychnos nux-vomica on human hepatoma cells is mediated via Bcl-2 and $\mathrm{Ca}^{2+}$ involved mitochondrial pathway. Toxicol Sci 91: 59-69, 2006.

7. Deng XK, Cai BC, Yin W, Liu TS, Sun Q and Li WD: Research of the anti-tumor effect and toxicity of brucine in Heps tumor-bearing mice. Chin Pharmacol Bulletin 22: 35-39, 2006 (In Chinese).

8. Deng XK, Cai BC, Yin W, Zhang XC, Li WD and Sun Q: Brucine on mouse tumor inhibition. Chin J Nat Med 3: 392-395, 2005 (In Chinese).

9. Qin JM, Zhang YD, Wang HY and Wu MC: Nanotechnology usage in diagnosis and treatment of liver diseases. Chin J Modern Med 13: 49-52, 2003 (In Chinese).

10. Qin JM, Zhang YD, Wang HY and Wu MC: Usage and progress of nanodrug in hepatic disease. Chin J Hepatobiliary Surg 10: 646-648, 2004 (In Chinese).

11. Gao Y, Li HL, Lv QZ and Zhai GX: Application of active transport path in targeting drug delivery system of liver. Chin J Pharmaceuticals 39: 542-547, 2008 (In Chinese).

12. Qin JM, Yin PH, Li Q, Sa ZQ, Sheng X, Yang L, Huang T, Zhang M, Gao KP, Chen QH, et al: Anti-tumor effects of brucine immuno-nanoparticles on hepatocellular carcinoma. Int J Nanomedicine 7: 369-379, 2012.

13. Gregoriadis G (ed): Liposome Technology. Vol 3. CRC Press, Boca Raton, FL, p75, 1984.

14. Hou XM, Cui LL, Li GD and Li WH: Advance on targeted drug delivery in cancer therapy. J Pharm Pract 25: 273-275, 2007.

15. Vasir JK and Labhasetwar V: Targeted drug delivery in cancer therapy. Technol Cancer Res Treat 4: 363-374, 2005.

16. Stefani M, Coudane J and Vert M: In vitro ageing and degradation of PEG-PLA diblock copolymer-based nanoparticles. Polymer Degradation Stability 91: 2554-2559, 2006.

17. $\mathrm{Lu} \mathrm{Y,} \mathrm{Li} \mathrm{J} \mathrm{and} \mathrm{Wang} \mathrm{G:} \mathrm{In} \mathrm{vitro} \mathrm{and} \mathrm{in} \mathrm{vivo} \mathrm{evaluation} \mathrm{of}$ mPEG-PLA modified liposomes loaded glycyrrhetinie acid. Int J Pharm 356: 274-281, 2008.

18. Park EK, Lee SB and Lee YM: Preparation and characterization of methoxy poly(ethylene glycol)/poly(epsilon-caprolactone) amphiphilic block copolymeric nanospheres for tumor-specific folate-mediated targeting of anticancer drugs. Biomaterials 26: 1053-1061, 2005.

19. Wei Q, Wei W, Tian R, Wang LY, Su ZG and Ma GH: Preparation of uniform-sized PELA microspheres with high encapsulation efficiency of antigen by premix membrane emulsification. J Colloid Interface Sci 323: 267-273, 2008.

20. van der Heiden PL, Jedema I, Willemze R and Barge RM: Efficacy and toxicity of gemtuzumab ozogamicin in patients with acute myeloid leukemia. Eur J Haematol 76: 409-413, 2006.

21. Adams GP and Weiner LM: Monoclonal antibody therapy of cancer. Nat Biotechnol 23: 1147-1157, 2005.

22. Wu AM and Senter PD: Arming antibodies: Prospects and challenges for immunoconjugates. Nat Biotechnol 23: 1137-1146, 2005.

23. Kan HP, Liu ZG, Tan YF, Lin YX, Li CF and Zhou J: Preparation of immune nanospheres of human liver cancer and observation of anti-cancer effect. J South Med Univ 28: 1503-1505, 2008 (In Chinese) 
24. Liu XB and Cai MY: Preparation of immune nanospheres of human liver cancer and identification of immunological properties in vitro. Chin J Immunol 16: 262-265, 2000 (In Chinese).

25. Li L: Study outline of effect and attenuation of brucine. Asia-Pacific Trad Med 5: 19-21, 2007 (In Chinese).

26. Wang L, Cai BC, Yang H, YU ZL, Deng XK and Zhang ZJ: A Comparative study on pharmacokinetics of vauqueline solution and vauqueline liposome in rabbits. J Nanjing TCM Univ 22: 165-167, 2006 (In Chinese).

27. Qu YQ, Guo YQ, Ha HX, Chen J and Cai BC: Comparison of the antitumor effect between brucine stealth liposome and brucine conventional liposome. J Trad Chin Drug Res Clin Pharmcol 19: 361-363, 2008 (In Chinese).

28. Wu J, Nantz MH and Zern MA: Targeting hepatocytes for drug and gene delivery: Emerging novel approaches and applications. Front Biosci 7: d717-725, 2002.

29. Kan HP, Tan YF, Li KF and Zhou J: Preparation and anticancer effects of immunonanospheres containing paclitaxel against human liver cancer. J Mod Dig Interven 15: 227-229, 2010 (In Chinese).
30. Hu KQ, Kyulo NL, Lim N, Elhazin B, Hillebrand DJ and Bock T: Clinical significance of elevated alpha-fetoprotein (AFP) in patients with chronic hepatitis $\mathrm{C}$, but not hepatocellular carcinoma. Am J Gastroenterol 99: 860-865, 2004.

31. Montaser LM, Abbas OM, Saltah AM and Waked IA: Circulating AFP mRNA as a possible indicator of hematogenous spread of HCC cells: A possible association with HBV infection. J Egypt Natl Cancer Inst 19: 48-60, 2007.

32. Kamiyama T, Takahashi M, Nakagawa T, Nakanishi K, Kamachi H, Suzuki T, Shimamura T, Taniguchi M, Ozaki M, Matsushita M, et al: AFP mRNA detected in bone marrow by real-time quantitative RT-PCR analysis predicts survival and recurrence after curative hepatectomy for hepatocellular carcinoma. Ann Surg 244: 451-463, 2006.

This work is licensed under a Creative Commons Attribution-NonCommercial-NoDerivatives 4.0 International (CC BY-NC-ND 4.0) License. 